\title{
Optimal Sizing of Distributed Energy in AC/DC Hybrid Stand-alone Micro-grid Using Modified Artificial Bee Colony Algorithm
}

\author{
Xingjie $\mathrm{Liu}^{1, \mathrm{a}}$, Zhe $\mathrm{Du}^{1, \mathrm{~b}}$ and Xiao Yan ${ }^{1, \mathrm{c}}$ \\ ${ }^{1}$ School of North China Electric Power University, Baoding 071003, China; \\ alxj5085@163.com, 'duzhew@126.com, ${ }^{\mathrm{c} 953692753 @ q q . c o m}$
}

Keywords: hybrid AC/DC micro-grid, control strategy, optimal sizing, modified artificial bee colony algorithm.

\begin{abstract}
In order to reduce investment and maintenance cost, the optimal sizing of distributed energy in AC/DC hybrid stand-alone micro-grid is studied in this paper. The optimization model is built and economics, power supply reliability and environmental protection are taken into account. By means of the modified artificial bee colony algorithm, the optimal sizing method is obtained. Finally, the feasibility of method is verified by calculating example analysis. By comparing the optimal sizing results of three different micro-grid configurations, hybrid AC/DC micro-grid is proved to be superior to traditional micro-grid. Furthermore, the modified ABC is more suitable for solving optimization problems because of the higher convergence precision.
\end{abstract}

\section{Introduction}

Hybrid AC/DC micro-grid combines with the advantage of AC micro-grid and DC micro-grid, and it can optimize the operation of DG(Distributed Generations), loads and ESS(Energy Storage System) [1]. Research on its optimal sizing can improve the utilization rate of renewable energy and reduce the investment and maintenance cost without sacrificing the reliability of micro-grid.

At present, Many experts and scholars have studied on optimal sizing of the DG and ESS in micro-grid [2-4]. However, due to the differences in system configuration and control strategy between AC/DC hybrid micro-grid and traditional micro-grid, the existed optimal sizing method of distributed energy in micro-grid cannot be directly applied in AC/DC hybrid micro-grid.

Based on the structure of AC/DC hybrid network containing ESS [5], supplementation and optimization of the existed control strategy [6] are carried out. The optimal sizing model is built with the objective that takes annual cost, environment protection and power supply reliability into account. By means of the modified ABC(Artificial Bee Colony) algorithm, which has strong capability of global research and rapid speed of convergence, the optimal sizing result for AC/DC hybrid stand-alone micro-grid is obtained.

In the end, the example demonstrates the feasibility of method. The comparative analysis indicates that the optimal sizing result of AC/DC hybrid stand-alone micro-grid is more economical.

\section{AC/DC hybrid stand-alone micro-grid system}

System configuration of AC/DC hybrid stand-alone micro-grid. In this paper, the system configuration of AC/DC hybrid stand-alone micro-grid is shown in Fig.1. It consists of three parts: AC subgrid, DC subgrid and storage battery system. There are four kinds of distributed energy: WT, $\mathrm{PV}$, storage batteries and diesel generators. Storage batteries are connected to DC bus. 


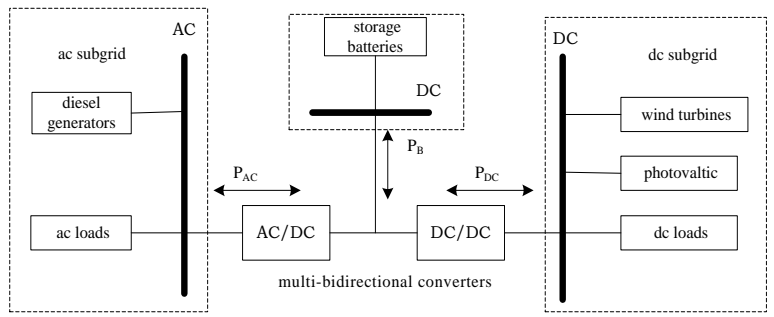

Fig.1 Structure of AC/DC hybrid stand-alone micro-grid

Control strategy of AC/DC hybrid stand-alone micro-grid. Based on the existed control strategy of AC/DC hybrid micro-grid [6], the improved control strategy is put forward as follows:

First, renewable energy generations are at the top of the priority list to supply power. Second, charging the batteries through the excess power of PV and MT or increase diesel generators' output when the SOC of batteries are not reach the upper limit.

The specific progress of control strategy is shown in Fig.2. Where $P_{1}$ is the sum output power of PV and WT; $P_{2}$ is the output power of single diesel generator; $n$ stands for the number of diesel generators which are already running; $n_{\max }$ stands for the total number of diesel generators; $P_{3}$ and $P_{4}$ are the charge and discharge power of storage batteries respectively; $P_{\mathrm{dc}}$ and $P_{\mathrm{ac}}$ are the loads of DC and AC subgrid respectively.

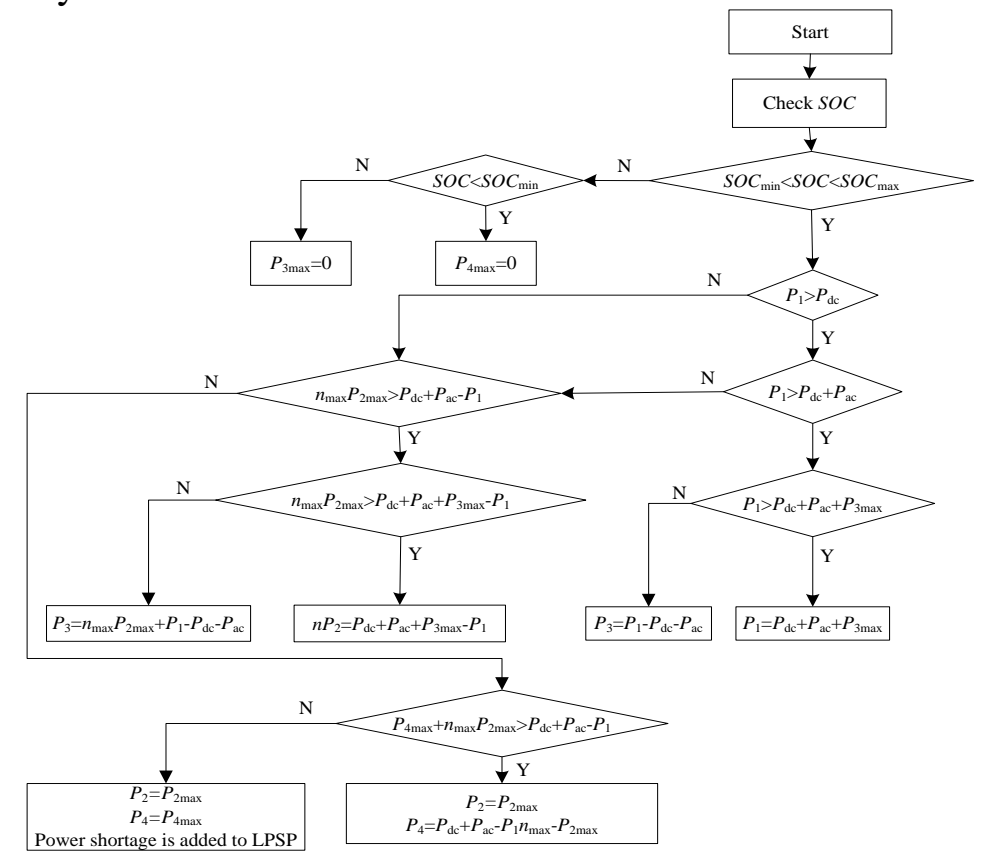

Fig.2 Improved operation strategy of AC/DC hybrid stand-alone micro-grid

In order to simplify the representation, the power transmission efficiency is omitted in Fig.2 and will be discussed further below.

\section{Optimal sizing model}

An optimal sizing model of distributed energy is presented with an economic objective, and the environmental protection is reduced to the objective function. The reliability index is considered in the form of a penalty function included in the objective function. The distributed energy optimal sizing is converted to a multi-constraint single-objective nonlinear integer programming problem.

Distributed energy model. The detailed distributed energy model are not reiterated here, the quasi-steady model of WT, PV and storage battery are presented respectively in References [7-9].

Multi-bidirectional converter model. Multi-bidirectional converter connects AC subgrid, DC subgrid and storage batteries together and controls three parts according to certain control strategy. The equation of inflow power to DC subgrid, AC subgrid and storage batteries $\left(P_{\mathrm{DC}}, P_{\mathrm{AC}}\right.$ and $\left.P_{\mathrm{B}}\right)$ is: 


$$
\begin{gathered}
P_{\mathrm{DC}} \eta_{1}+P_{\mathrm{AC}} \eta_{2}+P_{\mathrm{B}}=0 \\
\eta_{1}=\left\{\begin{array}{c}
1 / \eta_{\mathrm{dc} \_\mathrm{dc}}, P_{\mathrm{DC}} \geq 0 \\
\eta_{\mathrm{dc} \_\mathrm{dc}}, P_{\mathrm{DC}}<0
\end{array}\right. \\
\eta_{2}=\left\{\begin{array}{c}
1 / \eta_{\mathrm{dc} \_\mathrm{ac}}, P_{\mathrm{AC}} \geq 0 \\
\eta_{\mathrm{ac} \_\mathrm{dc}}, P_{\mathrm{AC}}<0
\end{array}\right.
\end{gathered}
$$

Where $\eta_{\text {dc_dc }}, \eta_{\text {dc_ac }}$ and $\eta_{\text {ac_dc }}$ are converter efficiency of DC/DC part, inversion and rectification efficiency of AC/DC part respectively.

Reliability of power supply model. LPSP (Loss of Power Supply Probability) is the measurement index which indicates the probability of power supply shortage.

$$
P_{\mathrm{LPSP}}=\frac{\sum_{t=1}^{8760} P_{\mathrm{LOSS}}(t)}{\sum_{t=1}^{8760} P_{\mathrm{LOAD}}}
$$

Where $P_{\text {LOAD }}(t)$ is total power of loads in micro-grid at $t$ moment ; $P_{\text {LOSS }}(t)$ is total shortage power of supply in micro-grid at $t$ moment.

Economic model. The objective function is the lowest annual average cost. The impact of diesel generators on environment is converted into cost and is added to the objective function as follow:

$$
\min C=\sum_{i=1}^{N}\left(C_{\mathrm{CP}_{i}}+C_{\mathrm{OM}_{i}}+C_{\mathrm{FC}_{i}}+C_{\mathrm{EC}_{i}}\right)+C_{\mathrm{IC}}
$$

Where $C_{\mathrm{CP} i}$ is equipment investment cost; $C_{\mathrm{OM} i}$ is operation and maintenance cost; $C_{\mathrm{FCi}}$ is fuel cost; $C_{\mathrm{EC} i}$ is environmental protection reduced cost. All of costs above are distributed energy $i$ 's equivalent annual value. $C_{\text {IC }}$ is investment, operation and maintenance cost of multi-bidirectional converter's annual cost. $N$ is the number of distributed energy types. Therefore, $N=4$.

Model constraints.

1) Output power constraints

$P_{i} \leq p_{i}^{\max } x_{i}$

Where $P_{i}$ is the output power of distributed energy $i ; p_{i}^{\max }$ is the maximum power per unit; $x_{i}$ is the number of units.

2) Storage batteries constraints

It is generally acknowledged that charge and discharge power per hour should not exceed $20 \%$ of its capacity. Besides, only when SOC is within certain realms can storage batteries charge or discharge.

$$
\begin{gathered}
\left\{\begin{array}{l}
P_{\mathrm{c}} \leq 0.2 E_{\text {bat }} / \Delta t \\
P_{\mathrm{d}} \leq 0.2 E_{\text {bat }} / \Delta t
\end{array}\right. \\
S O C_{\text {min }} \leq S O C \leq S O C_{\text {max }}
\end{gathered}
$$

Where $\Delta t=1 \mathrm{~h}$.

3) Reliability constraints

$P_{\mathrm{LPSP}} \leq P_{\mathrm{SET}}$

Where $P_{\text {SET }}$ is the specified loss power supply probability in micro-grid.

Optimization variables. The types of PV, WT, storage battery and diesel generator are determined. Therefore, the optimization variables are changed form the sizing of distributed energy to the number of them. The optimization variables are defined as follow:

$X=\left[N_{\mathrm{WTG}}, N_{\mathrm{PV}}, N_{\mathrm{BAT}}, N_{\mathrm{D}}\right]$

\section{Modified artificial bee colony algorithm}


ABC algorithm is a novel intelligence optimization algorithm introduced by Karaboga in 2005. $\mathrm{ABC}$ mainly simulates the intelligent foraging behavior of bee colony. $\mathrm{ABC}$ has the advantages of less control parameters, higher search veracity and stronger robustness [10,11].

First, randomly create distributed initial population, that is initial solutions. Each solution $x_{i}(i=1,2 \ldots S N)$ is a $D$-dimensional vector, where $D$ is the number of parameters to be optimized; $S N$ is the number of employed bees and also the number of food-source.

Employed bees create a new candidate food-source position according to original position and check the nectar amounts of the new position. They remember the position with more nectar amounts.

The modified search equation of ABC algorithm is proposed referring to differential evolution algorithm [12]. The new candidate solution is obtained in new search region which nears the optimal solution of last iteration. In this way, the convergence speed is improved through the direction of optimal solution in searching food-source. The global search capability is retained by the difference between two solutions which are randomly selected.

$v_{i j}=x_{\text {best }, j}+\phi_{i j}\left(x_{\mathrm{r} 1, j}-x_{\mathrm{r} 2, j}\right)$

Where $x_{\text {best }, j}$ is the optimal solution of last iteration; $x_{\mathrm{r} 1}$ and $x_{\mathrm{r} 2}$ are two random solutions; $\varphi_{i j}$ is the random number between $[-1,1], j \in\{1,2, \cdots D\}$.

Onlooker bees choose a food-source position with a probability related to its nectar amount, then change the position in their memory.

The probability of selecting a food-source $p_{i}$, that is transfer probability is calculated as follows:

$$
p_{i}=\frac{f i t_{i}}{\sum_{n=1}^{S N} f i t_{n}}
$$

Where fit $_{i}$ is the fitness value of solution $x_{i}$.

If the food-source position does not improve for a specified number of times, it will be abandoned. The scout bee randomly search a food-source position to replace the previous one. The search equation of scout bee is:

$$
x_{i}^{j}=x_{\min }^{j}+\operatorname{rand}(0,1)\left(x_{\max }^{j}-x_{\min }^{j}\right)
$$

Where $x_{\max }^{j}$ and $x_{\max }^{j}$ are upper and lower bound of search space respectively.

The flowchart of the modified ABC algorithm is illustrated in Fig.3.

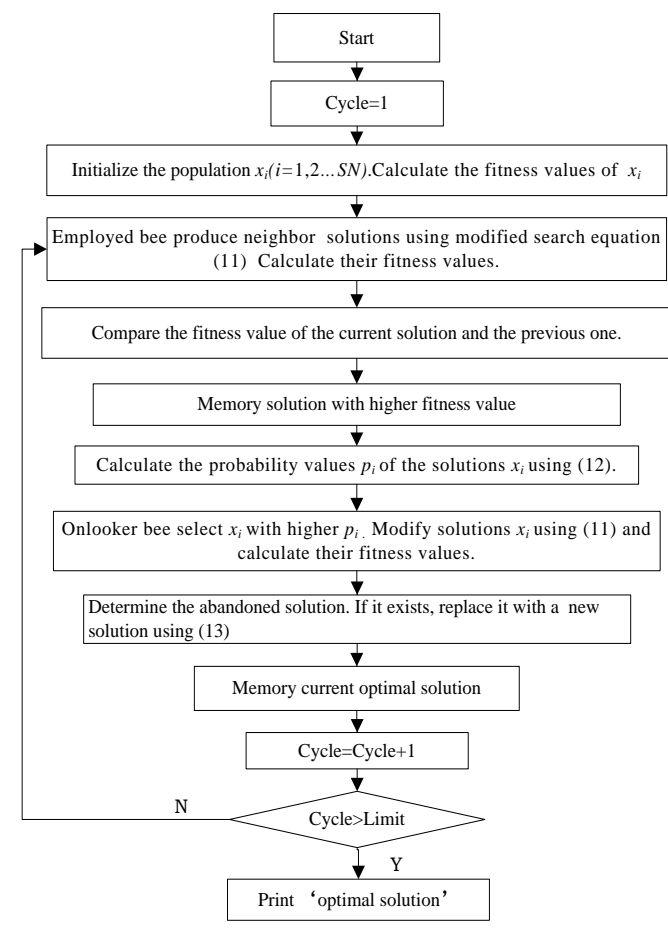

Fig.3 Calculation flow chart of modified artificial bee colony algorithm 


\section{Example analysis}

Calculating the optimal sizing problem via Matlab. The simulation time is $T=1 \mathrm{a}$, simulation step size is $1 \mathrm{~h}$, construction period is $13 \mathrm{a}$, discount rate is $r=6.7 \%$. Suppose maximum allowed loss power supply probability is $0.1 \%$, which means the power supply reliability is $99.9 \%$.

The annual average AC and DC loads are $105 \mathrm{~kW}$ and $193 \mathrm{~kW}$ respectively. The annual loads curve, annual meteorological data including light intensity, wind speed and temperature, and related parameters of distributed energy are taken as the input data. The specific parameters of distributed energy is indicated in Tab.1.

Tab.1 Unit capacity and related costs of distributed energy

\begin{tabular}{ccccccc}
\hline DG & $\begin{array}{c}\text { Unit } \\
\text { capacity } \\
(\mathrm{kW})\end{array}$ & $\begin{array}{c}\text { Unit cost } \\
(¥ / \mathrm{kW})\end{array}$ & $\begin{array}{c}\text { Installation } \\
\text { cost } \\
(¥ / \mathrm{kW})\end{array}$ & $\begin{array}{c}\text { Operation and } \\
\text { maintenance cost } \\
(¥ /(\mathrm{kW} \cdot \mathrm{a}))\end{array}$ & $\begin{array}{c}\text { Fuel cost } \\
(¥ /(\mathrm{kW} \cdot \mathrm{h}))\end{array}$ & $\begin{array}{c}\text { Environmental } \\
\text { protection cost } \\
(¥ /(\mathrm{kW} \cdot \mathrm{h}))\end{array}$ \\
\hline Wind turbine & 10 & 11730 & 8130 & 37.05 & 0 & 0 \\
Photovoltaic & 8.3 & 14370 & 6550 & 92.95 & 0 & 0 \\
Storage battery & $50(\mathrm{~kW} . \mathrm{h})$ & 7800 & 1300 & 45.50 & 0 & 0 \\
Diesel generator & 60 & 3840 & 3090 & 172.25 & 2.176 & 0.067 \\
\hline
\end{tabular}

Comparative analysis of AC/DC hybrid micro-grid and traditional micro-grid.The optimal sizing results of three kinds of micro-grid configurations are obtained under the same loads and meteorological conditions and the same control strategy. In order to simplify the analysis, the equipment voltage is consistent with bus voltage in three schemes, and the rectification and inversion efficiency are supposed to be 96\%, the cost of rectifier and inverter in AC micro-grid and DC micro-grid is $800 ¥ / \mathrm{kW}$.

The optimal sizing results of three kinds of micro-grid configurations are shown in Tab.2. Scheme 1 to 3 correspond to AC micro-grid, DC micro-grid and AC/DC hybrid micro-grid respectively.

Tab.2 Optimal sizing results of three different micro-grid configurations

\begin{tabular}{ccccccc}
\hline Scheme & $\begin{array}{c}\text { WT } \\
\text { (per } \\
\text { unit) }\end{array}$ & $\begin{array}{c}\text { PT } \\
\text { (per } \\
\text { unit) }\end{array}$ & $\begin{array}{c}\text { Storage } \\
\text { battery } \\
\text { (per } \\
\text { unit) }\end{array}$ & $\begin{array}{c}\text { Diesel } \\
\text { generator } \\
\text { (per unit) }\end{array}$ & $\begin{array}{c}\text { Total } \\
\text { equivalent } \\
\text { annual } \\
\text { cost( } ¥)\end{array}$ & $\begin{array}{c}\text { Annual } \\
\text { converter } \\
\text { loss(kW·a) }\end{array}$ \\
\hline 1 & 48 & 69 & 0 & 7 & 611600 & 19.741 \\
2 & 50 & 75 & 1 & 6 & 589600 & 9.054 \\
3 & 39 & 90 & 31 & 3 & 472700 & 4.356 \\
\hline
\end{tabular}

The table above indicates that the total equivalent annual cost and annual converter loss from high to low, in order, is AC micro-grid, DC micro-grid and AC/DC hybrid micro-grid. This result conforms to the actual situation that a high share of DC loads in system and the DC power, such as WT and PV, and storage battery can be conveniently integrated into DC micro-grid. The cost in AC micro-grid is highest because DC power and DC loads increase the investment and loss cost of converter.

In AC/DC hybrid micro-grid, power and loads are connected to the relevant side, which reduces the cost of converter links and power electronic devices. Furthermore, the number of diesel generator in AC/DC hybrid micro-grid is less than that in traditional micro-grid, nevertheless, the reliability of power supply can be ensured due to the increase of storage battery. The impact of diesel generator on environment is also reduced, which means the AC/DC hybrid micro-grid is more environmentally friendly than traditional micro-grid.

Comparative analysis of modified ABC and other algorithms. The convergence curves shown in Fig.4 indicates that though ABC's convergence speed is slightly inferior to GA, its convergence precision is superior to GA. The modified ABC improves the convergence speed while maintaining convergence precision. As the precision is more essential for the optimal sizing in micro-grid, the modified ABC has a major practical significance. 


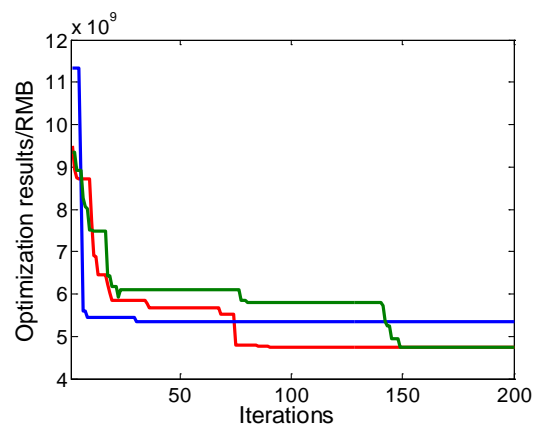

Fig.4 Calculation results of ABC, GA and modified ABC

\section{Conclusions}

In this paper, the optimal sizing of distributed energy in AC/DC hybrid stand-alone micro-grid is studied. An optimal sizing model is presented with an economic objective, in addition, environmental protection and power supply reliability are taken into account. The optimal solution is obtained using modified ABC algorithm. The example analysis verifies the feasibility of optimal sizing method, and the main conclusions are as follows:

(1) Comparing with traditional AC or DC micro-grid, power and loads are connected to the relevant side in AC/DC hybrid micro-grid, which reduces the cost of converter links and power electronic devices. The results show that AC/DC hybrid micro-grid is more economical.

(2) Storage battery system is preferred for maintaining the unbalance between power and load. The increase of storage batteries has reduced the number of diesel generators. In that case, the reliability of micro-grid operation is guaranteed and the environment pollution is reduced.

(3) ABC algorithm has higher convergence precision than traditional intelligence algorithms. The modified ABC improves the convergence speed, therefore, it is more suitable for solving nonlinear integer programming problems.

\section{References}

[1] Guerrero J M, Vasquez J C, Matas J, et al. Hierarchical control of droop-controlled AC and DC microgrids-A general approach toward standardization[J]. Industrial Electronics, IEEE Transactions on, 2011, 58(1): 158-172.

[2] Ma X, Wu Y, Fang H, et al. Optimal sizing of hybrid solar-wind distributed generation in an islanded microgrid using improved bacterial foraging algorithm[J]. Proceedings of the Chinese Society for Electrical Engineering, 2011, 31(25): 17-25.

[3] Chen J, Wang C, Zhao B, et al. Optimal sizing for stand-alone microgrid considering different control strategies[J]. Automation of Electric Power Systems, 2013, 37(11): 1-6.

[4] Guo L, Liu W J, Jiao B Q. Multi-objective optimal planning design method for stand-alone microgrid system[J]. Proceedings of the CSEE, 2014, 34(4): 524-536.

[5] Loh P C, Li D J, Chai Y K, et al. Hybrid AC-DC microgrids with energy storages and progressive energy flow tuning[J]. Power Electronics, IEEE Transactions on, 2013, 28(4): 1533-1543.

[6] Wang P, Jin C, Zhu D, et al. Distributed control for autonomous operation of a three-port AC/DC/DS hybrid microgrid[J]. Industrial Electronics, IEEE Transactions on, 2015, 62(2): 1279-1290.

[7] Ning Y B, Zheng J Y, Xia L M, et al. Research and analysis on comprehensive output characteristics of photovoltaic power stations[J]. Acta Energiae Solaris Sinica, 2015, 36(5):1197-1205. 
[8] Zhang J T, Chen H Z, Hu Z C, et al. Power system probabilistic production simulation including wind farms[J]. Proceedings of the CSEE, 2009, 29(28): 34-39.

[9] Xing L. Modeling and simulation of Multi-objective optimal operation and energy management for microgrid[D]. Shanghai Jiao Tong University, 2013.

[10] Abu-Mouti F S, El-Hawary M E. Optimal distributed generation allocation and sizing in distribution systems via artificial bee colony algorithm[J]. Power Delivery, IEEE Transactions on, 2011, 26(4): 2090-2101.

[11]Karaboga D, Akay B. A comparative study of artificial bee colony algorithm[J]. Applied mathematics and computation, 2009, 214(1): 108-132.

[12] Yu S W. MATLAB optimization algorithm of case analysis and application: advanced[M]. Beijing: Tsinghua University Press, 2015: 465-474. 